\title{
EL MEDITERRÁNEO ORIENTAL Y LA DIVERSIFICACIÓN DE GAS A EUROPA
}

\begin{abstract}
Antonio SÁNCHEZ ORTEGA*
SUMARIO: 1. INTRODUCCIÓN.-2. EL ABASTECIMIENTO DE GAS EN LA UE.-3. EL MEDITERRÁNEO ORIENTAL Y SU POTENCIAL GASÍSTICO.-4. CONCLUSIONES.
\end{abstract}

\section{INTRODUCCIÓN}

1. La Unión Europea (UE), cuando menos, presenta una situación complicada en lo que respecta al abastecimiento de gas natural. Dicha complejidad radica en tres elementos principales. En primer lugar su alta dependencia exterior. La UE es el primer importador de gas a nivel global, pero además el descenso de su producción interna va a provocar un incremento de la dependencia exterior. En segundo lugar, la excesiva concentración de sus importaciones. Esto ha sido especialmente traumático en el caso de Rusia. Desde que en 2006 comenzaran a producirse una serie de crisis políticas entre Ucrania y Rusia, la dependencia energética de Moscú ha sido identificada como uno de los principales riesgos que ha de enfrentar la Política Energética Europea. En tercer lugar, las diferentes iniciativas que han tenido como objetivo incrementar la diversificación de las importaciones de gas no han dado los resultados esperados. Es por ello que los recientes descubrimientos de importantes yacimientos gasísticos en el Mediterráneo oriental están despertando un creciente interés en Europa.

\section{EL ABASTECIMIENTO DE GAS EN LA UE}

2. Los datos de la dependencia de gas de la UE servirían de ejemplo para ilustrar un manual de seguridad energética. En la actualidad, las importacio-

\footnotetext{
* Profesor Ayudante Doctor de Derecho Internacional Público y Relaciones Internacionales de la Universidad de Granada (santega@ugr.es). Todos los enlaces a sitios web han sido comprobados con fecha de 30 de octubre de 2018.
} 
nes de gas suponen el 70 por 100 del total. El descenso de la producción endógena y el aumento del consumo llevarán esta dependencia al 87 por 100 para $2050^{1}$. Esto es aún más problemático si tenemos en cuenta que existe una alta concentración de las fuentes de suministro. Rusia, Noruega y Argelia proporcionan el 77 por 100 de las importaciones de gas natural a la UE. Además, Rusia con el 40 por 100 del abastecimiento de gas destaca como el principal abastecedor. Los problemas y temores de esta alta dependencia de Rusia han sido bien documentados y han provocado verdaderos quebraderos de cabeza por las crisis entre este país y Ucrania, la principal vía de transporte de gas a Europa, en 2006 y 2009 que afectaron al abastecimiento europeo y, especialmente, en 2014, tras la anexión de Crimea por parte de Rusia. Esta crisis provocó la aprobación de sanciones contra Rusia por parte de la UE que, entre otros sectores, afectan a su industria energética. Sin duda, no es posible desligar estas situaciones de la creación de la Política Energética Europea ${ }^{2}$.

3. Una de las acciones más perseguidas por las Instituciones Europeas para mejorar el aprovisionamiento de la Unión ha pasado por incrementar la diversificación del aprovisionamiento. En la actualidad, los gasoductos con destino a la UE están diversificados en tres corredores: Corredor Septentrional desde Noruega, Corredor Oriental desde Rusia y Corredor Mediterráneo desde África. Por tanto, sus esfuerzos se han dirigido a desarrollar un nuevo eje de abastecimiento, el Corredor Meridional. Este corredor se concibió para unir directamente la UE con la cuenca del mar Caspio y Oriente próximo, donde se encuentran más del 49 por 100 de las reservas de gas - frente al 17 por 100 de Rusia- y se pensó que podría aportar entre el 10-20 por 100 del consumo europeo para 2020. La concreción material del Corredor Meridional ha pasado por diferentes proyectos de gasoductos, prevaleciendo en la actualidad el TAP, que conectaría los yacimientos de gas de Azerbaiyán con el sudeste europeo ${ }^{3}$. A pesar de la creación de este gasoducto, es necesario reconocer que este no cumple las expectativas originales del corredor meridional. En el mejor de los casos, el TAP, supondría el aporte de 10.000 millones de metros cúbicos (mmmc) anuales, lo que solo supone un 2 por 100 del consumo actual de gas de la UE y aunque se ha planteado su ampliación, e incluso su conexión con Turkmenistán, parece poco probable que pueda incrementar su aportación de manera significativa ${ }^{4}$.

\footnotetext{
1 European Commission, EU Energy, Transport and GHG Emissions - Trends To 2050_, EU Reference Scenario, 2016, p. 72.

2 Véase Alp KocaK, K., De Micco, P. y Felici, A., «The quest for natural gas pipelines - EU and Eastern Partner energy policies: Security versus transit benefits", EPRS European Parliamentary Research Service and Directorate General for External Policies, julio de 2016.

3 Aunque nos referimos a él como TAP, lo cierto es que son tres gasoductos conectados entre sí. El TAP entre Italia, Albania y Grecia, el TANAP — Trans Anatolian Natural Gas Pipeline—, a través de Turquía y el SCP — South Caucasus Pipeline- que conecta con los yacimientos de gas en Azerbaiyán. Cuando esté terminado, para 2019, transportará 16.000 millones de metros cúbicos anuales, de los cuales seis mil serán para Turquía y diez mil para los mercados de Grecia e Italia. Véase BP, Shah Deniz 2 and Opening of the Southern Corridor, disponible en http://www.bp.com/content/dam/bp/pdf/ Press/shah_deniz_2_brochure_english.pdf.

4 Se ha previsto una ampliación de su capacidad hasta los $20 \mathrm{mmmc}$ anuales más allá de 2020, opción que ha cobrado fuerza tras la crisis entre Ucrania y Rusia. HAFNER, M., «The southern gas corridor
} 
4. Es por ello que, en la actualidad, las circunstancias técnicas y los desafíos de carácter geopolítico parecen haber dado al traste con la mayor incitativa de diversificación de la UE. Desde que el proyecto nació, hace más de quince años y a pesar de la atención e interés mostrado por la UE, el corredor meridional parece encontrarse en un callejón sin salida, en cambio, los recientes descubrimientos de gas en el Mediterráneo oriental podrían insuflar algo de vida a este proyecto.

\section{EL MEDITERRÁNEO ORIENTAL Y SU POTENCIAL GASÍSTICO}

5. En la cuenca geográfica que va desde la costa sur de Turquía hasta la desembocadura del Nilo se han realizado importantes descubrimientos de gas natural que podrían llegar a exceder las reservas de Argelia, uno de los principales suministradores de gas a Europa ${ }^{5}$. Por cuestiones tanto de carácter político, conflictos u otros problemas de carácter interno, como técnico, cuantía de las reservas y desarrollo de la industria gasística, los yacimientos más prometedores, desde el punto de su potencial exportador, se han descubierto en Egipto, Chipre e Israel.

6. De entre ellos destacan los yacimientos de Egipto. Desde el año 1999 se han realizado importantes descubrimientos de gas en el país árabe, acumulando en total $1.800 \mathrm{mmmc}$, casi el 1 por 100 de las reservas mundiales de gas $^{6}$. Además, cuenta con una industria gasística bastante desarrollada, que incluye dos terminales para la exportación de gas natural licuado (GNL) y el gasoducto árabe que permite exportar gas a Jordania, Israel, Siria y Líbano. Todo ello le ha permitido ser el segundo productor de gas en África, por detrás de Argelia y el décimo quinto exportador de gas mundial. Sin embargo, el incremento del consumo endógeno, la falta de inversión y los problemas políticos internos lo convirtieron en importador de gas en 2015. Se espera que los nuevos yacimientos y los cambios legislativos que persiguen, por un lado, la atracción de capital exterior y, por otro, la mayor eficiencia del consumo interno permitan a Egipto volver a exportar gas en 2021. Condición

and the EU gas security of supply: what's next?», Natural Gas World, 28 de marzo de 2015, disponible en https://www.naturalgasworld.com/southern-gas-corridor-and-eu-gas-security-of-supply-22688. A pesar de lo cual, existen dudas de que esto pueda lograrse. Véase PIRANI, S., «Azerbaijan's gas supply squeeze and the consequences for the Southern Corridor», Oxford Institute for Energy Studies Paper, NG 110, julio de 2016, y PIRANI, S., "Let's not exaggerate: Southern Gas Corridor prospects to 2030 », Oxford Institute for Energy Studies Paper, NG 135, julio de 2018.

5 En total se han descubierto reservas por un total de tres billones de metros cúbicos, aunque podrían suponer más del doble. En su mayor parte se concentran en Egipto, $1.800 \mathrm{mmmc}$, estando el resto, 1.200, repartidos entre Chipre, Siria, Libano e Israel. Véase KARBUZ, S., «Geostrategic Importance of East Mediterranean Gas Resource», en Dorsman, A. et al. (eds.), Energy Economy, Finance and Geostrategy, Berlín, Springer, 2018, pp. 237-255, y Yorucu, V. y Mehmet O., «Energy Actors in the Eastern Mediterranean: Maps and Rivalries», en Vedat Yorucu, V. y Mehmet, O., The Southern Energy Corridor: Turkey's Role in European Energy Security. Lecture Notes in Energy, vol. 60, Berlín, Springer, 2018, pp. 103-118.

6 BP, BP Statistical Review of World Energy, junio de 2018. 
que podría mantener durante un periodo inferior a diez años con volúmenes superiores a $10 \mathrm{mmmc}$ anuales ${ }^{7}$.

7. Israel por su parte, salvo un breve periodo de tiempo entre 2004 y 2008, en el que llegó a producir el 40 por 100 del gas que consumía, ha sido tradicionalmente un país importador de gas. Los descubrimientos, en 2009 y 2010, de los inmensos yacimientos de Tamar y Leviatán ${ }^{8}$, entre otros, y su puesta en funcionamiento pueden convertir a Israel en exportador de gas en el corto plazo $^{9}$. El tercer Estado identificado en nuestro análisis es Chipre. A pesar de ser también un importador de gas, en los últimos años se han realizado descubrimientos, principalmente el yacimiento Afrodita, al sur de su zona económica exclusiva, con lo que se excluyen los problemas de demarcación territorial con la República Turca del Norte de Chipre. Las previsiones sitúan la capacidad de producción de Afrodita en torno a los 8,2 mmmc anuales, que en su mayor parte se podrían dedicar a la exportación. En ambos casos, Israel y Chipre, continúan las exploraciones en busca de nuevos yacimientos, con lo que no es descabellado pensar que pudieran incrementar sus reservas. A ellos se les podría unir Líbano que, a pesar de los delicados equilibrios políticos internos, está avanzando en el desarrollo de su sector gasístico.

8. Teniendo en cuenta el potencial de la región, sería conveniente analizar cuánto de ese gas podría llegar a Europa y cuál serían las vías más probables para su transporte. Es necesario indicar que todos los trabajos analizados tienden a presentar los intereses de exportación de Chipre e Israel vinculados de alguna forma, ya sea por la situación y proximidad geográfica de los mismos, como por la peculiar situación geopolítica de ambos actores en la región.

9. Desde que comenzaron a realizarse los primeros descubrimientos en la región, las autoridades egipcias, apoyadas incluso por el Banco Mundial y la UE, han trabajado intensamente para convertir a Egipto en un centro para la recepción del gas de la región ${ }^{10}$. Esta vía tiene importantes ventajas. En primer lugar hay que destacar que Egipto es un gran mercado para el gas natural y aunque puede convertirse en exportador en la próxima década, las previsiones indican que volverá a importar gas en el medio plazo. En segundo lugar y, como hemos comentado, cuenta con una desarrollada industria gasística. Así, podría exportar más de $16 \mathrm{mmmc}$ anuales mediante GNL y otros $19 \mathrm{mmmc}$ por gasoducto. Desde que se convirtió en importador, en 2015,

\footnotetext{
7 Véase OUKI, M., «Egypt - a return to a balanced gas market?», OIES PAPER, Oxford Institute for Energy Studies, NG 131, 2018, pp. 4 y ss.

8 Estos han sido catalogados como los mayores yacimientos de gas descubiertos en aguas profundas en el siglo XXI. Se estima que las reservas de ambos exceden los 970 mmmc. Véase KARBUZ, S., op. cit., nota 5, pp. 239-240.

9 Existe un acuerdo político para dedicar el 40 por 100 de las reservas de gas del país a la exportación. RuBLE, I., «European Union energy supply security: The benefits of natural gas imports from the Eastern Mediterranean», Energy Policy, 2017, núm. 105, pp. 341-353, esp. p. 346.

10 Véase Prontera, A. y Ruszel, M., "Energy Security in the Eastern Mediterranean», Middle East Policy, vol. 24, 2017, núm. 3, pp. 145-162.
} 
estas infraestructuras se encuentran infrautilizadas. Es por ello que desde el punto de vista económico la opción de convertir a Egipto en el centro de destino o de distribución del gas de la región parece la opción más viable. Esta situación ha provocado que las autoridades de Egipto, Chipre e Israel hayan discutido y llegado a algunos acuerdos preliminares de importación de gas israelí y para la creación de un gasoducto desde los yacimientos chipriotas a Egipto ${ }^{11}$. Sin embargo, a pesar de sus innegables ventajas, esta ruta cuenta con problemas de carácter político, especialmente en lo que respecta a la relación entre Egipto e Israel, que pueden acabar afectando a la colaboración entre Chipre y Egipto en materia de gas. Así, el gasoducto entre Egipto e Israel ha sido objetivo de ataques terroristas, a la vez que muy criticado por sectores de la población egipcia, mientras que, a pesar de las estables relaciones diplomáticas entre ambos, en ocasiones, estas atraviesan por fases de tensión política. Además, la propia inestabilidad interna de Egipto de los últimos años también plantea ciertas incertidumbres ${ }^{12}$.

10. Israel y Chipre han estudiado otras opciones para la exportación de gas. Principalmente estas serían mediante la creación de instalaciones de GNL en sus respectivos territorios, lo que ha sido desechado por cuestiones de seguridad en el caso israelí y por su alto coste en el chipriota. La exportación de gas al corredor meridional y su posible conexión con el TAP también quedan excluidos puesto que esto supondría la creación de un gasoducto entre ambos y Turquía. A pesar de los importantes beneficios económicos que tendrían para las partes, no parece que la situación política lo permita. Por último, los mercados jordano y palestino no parecen la opción prioritaria para los israelíes.

11. Sin duda, la opción que, hasta la fecha, ha suscitado un mayor entusiasmo político es la creación de un gaseoducto que comunique el Mediterráneo oriental con Europa. El East Med, como se ha denominado al proyecto, tendría más de 2.000 kilómetros y uniría los yacimientos chipriotas con el sur de Italia a través de Creta y Grecia. Esto lo libraría de los problemas derivados de los Estados de tránsito que han afectado a los suministros rusos y mejoraría la seguridad energética de Creta y Grecia que están aislados energéticamente. Su construcción, si finalmente se aprueba, está prevista para 2024 y podría transportar hasta $14 \mathrm{mmmc}$ anuales, aunque en una segunda fase podría aumentar hasta los 20 , para lo que sería necesario su

11 En el caso de Israel, el gas transitaría por los gasoductos existentes entre ambos países. El contenido último de los acuerdos no se ha hecho público, lo que sí se sabe es que el gas llegaría a Egipto, pero se desconoce si este se reexportaría a otros destinos distintos del mercado egipcio. Véase TsAKIRIS, T., «Cyprus's Natural Gas Strategy: Geopolitical and Economic Preconditions», Mediterranean Quarterly, 2017, núm. 28 (1), pp. 29-57.

12 A pesar de lo dicho, lo cierto es que los importantes descubrimientos de gas en la región y mediante la actuación de Grecia y Chipre, Israel y Egipto han acercado posturas en detrimento de la influencia de Turquía en la región. Véase KaragianNIS, E., "Shifting Eastern Mediterranean Alliances», Middle East Quarterly, vol. 23, 2016, núm. 2; y ADAMIDES, C. y CHRISTOU, O., «Energy Security and the Transformation of Regional Securitization Relations in the Eastern Mediterranean», en KATSIKIDES, S. y KoKtsidis, P. (eds.), Societies in Transition, Berlín, Springer, 2015, pp. 189-205. 
conexión con Israel. El East Med, cuenta con un importante apoyo político, tanto entre de las Instituciones Europeas como entre las autoridades de Chipre e Israel ${ }^{13}$. El entusiasmo suscitado por este proyecto no va necesariamente de la mano de la viabilidad económica, siendo este uno de los retos a los que debe hacer frente. A pesar de que el gas que entregaría podría tener un coste ligeramente superior al del gas ruso o incluso al GNL ${ }^{14}$, lo cierto es que las importantes ventajas que tendría como son aumentar la diversificación energética de la UE; reforzar el corredor meridional; incrementar la liquidez del mercado gasista europeo y la mayor interconexión de regiones energéticamente aisladas de Europa, podrían ser un importante elemento en su favor.

12. Por último, un aspecto clave que nos puede ayudar a entender la importancia que los yacimientos gasísticos descubiertos en el Mediterráneo oriental pueden tener en la diversificación del gas europeo, es el interés mostrado por Rusia para que sus empresas puedan participar en la explotación de los mismos. El objetivo último de dicha participación sería, además del económico, el de poder condicionar el destino final de esos recursos y evitar que puedan convertirse en una competencia para los rusos. Es necesario señalar que, salvo en los yacimientos egipcios y $\operatorname{sirios}^{15}$, sus aspiraciones no se han visto satisfechas, optando tanto Israel como Chipre por la participación mayoritaria de empresas occidentales ${ }^{16}$.

\section{CONCLUSIONES}

13. En 2017 la UE importó más de 190 mmmc de gas de Rusia. Esta dependencia del gas ruso no ha dejado de crecer a pesar de las múltiples crisis políticas entre las partes. En el mejor de los casos, el corredor meridional y los nuevos descubrimientos del Mediterráneo oriental podrían entregar de manera conjunta algo más de $30 \mathrm{mmmc}$ anuales en las próximas décadas. Teniendo en cuenta el incremento del consumo de la UE y el descenso en su producción interna, los nuevos aportes de gas, en ningún caso, servirían para reducir la dependencia de Rusia. Sí, en cambio, podrían contribuir a que esta no incrementase. Es por ello que parece claro que, en el actual contexto geopolítico, la diversificación del gas europeo no va a producirse mediante la

13 La UE lo ha declarado proyecto de interés común por lo que se hace cargo del 50 por 100 de la financiación de los gastos de estudio técnico para el desarrollo del proyecto. Véase EUROPEAN Commission, EastMed Pipeline Project - Development Phase, disponible en https:/lec.europa.eu/inea/en/ connecting-europe-facility/cef-energy/7.3.1-0023-cyel-s-m-17, y «Cyprus, Israel, Greece Push for Gas Pipeline to Europe», Pipeline and Gas Journal, 5 de agosto de 2018, disponible en https://pgionline.com/ news/2018/05/cyprus-israel-greece-push-for-gas-pipeline-to-europe.

14 Véase Ruble, I., op. cit., nota 9, p. 349.

15 Véanse OuKI, M., op. cit., nota 7, p. 9, y RUBLE, I., op. cit., nota 9, p. 351.

16 Véanse Shaffer, B., «Israel. New natural gas producer in the Mediterranean», Energy Policy, 2011, núm. 39, pp. 5379-5387, esp. p. 5386; PronTERA, A. y Ruszel, M., op. cit., nota 10, y STERGIOU, A., "Russia's Energy and Defense Strategy in the Eastern Mediterranean», Economics World, vol. 5, 2017, núm. 2, pp. 101-119. 
interconexión con nuevos yacimientos, por lo que, sin duda, es en el mercado creciente del GNL donde la UE podría encontrar el gas necesario para reducir su dependencia gasística de Rusia. Para que esto sea posible, la UE debe favorecer la interconexión de los gasoductos internos, cuestión que a veces se ha planteado tan ardua como la creación de gasoductos en el exterior.

Palabras clave: seguridad energética, Unión Europea, gas natural, Mediterráneo oriental.

Keywords: Energy security, European Union, natural gas, eastern Mediterranean. 